This article was downloaded by: [Jean-Claude J olly]

On: 26 September 2013, At: 08: 39

Publisher: Taylor \& Francis

Informa Ltd Registered in England and Wales Registered Number: 1072954 Registered

office: Mortimer House, 37-41 Mortimer Street, London W1T 3J H, UK

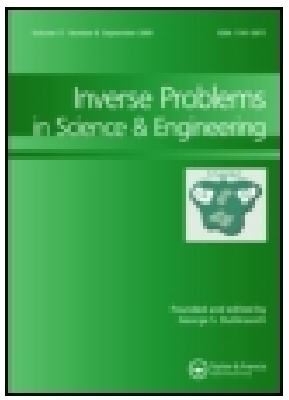

\title{
Inverse Problems in Science and Engineering
}

Publication details, including instructions for authors and subscription information:

http:// www.tandfonline.com/loi/ gipe20

\section{An inverse geometry problem for a one-dimensional heat equation: advances with complex temperatures}

\author{
J ean-Claude J olly ${ }^{a}$, Laetitia Perez ${ }^{b} \&$ Laurent Autrique ${ }^{a}$ \\ a Laboratoire d'Ingénierie des Systèmes Automatisés, EA 4094, \\ Université d'Angers-ISTIA, Angers, France. \\ b Laboratoire de Thermocinétique de Nantes, UMR 6607, Ecole \\ Polytechnique de l'Université de Nantes, Nantes Cedex 3, France. \\ Published online: 02 Sep 2013.
}

To cite this article: J ean-Claude J olly, Laetitia Perez \& Laurent Autrique, Inverse Problems in Science and Engineering (2013): An inverse geometry problem for a one-dimensional heat equation: advances with complex temperatures, Inverse Problems in Science and Engineering, DOI: 10.1080/ 17415977.2013.827186

To link to this article: http:// dx.doi.org/ 10.1080/ 17415977.2013.827186

\section{PLEASE SCROLL DOWN FOR ARTICLE}

Taylor \& Francis makes every effort to ensure the accuracy of all the information (the "Content") contained in the publications on our platform. However, Taylor \& Francis, our agents, and our licensors make no representations or warranties whatsoever as to the accuracy, completeness, or suitability for any purpose of the Content. Any opinions and views expressed in this publication are the opinions and views of the authors, and are not the views of or endorsed by Taylor \& Francis. The accuracy of the Content should not be relied upon and should be independently verified with primary sources of information. Taylor and Francis shall not be liable for any losses, actions, claims, proceedings, demands, costs, expenses, damages, and other liabilities whatsoever or howsoever caused arising directly or indirectly in connection with, in relation to or arising out of the use of the Content.

This article may be used for research, teaching, and private study purposes. Any substantial or systematic reproduction, redistribution, reselling, loan, sub-licensing, systematic supply, or distribution in any form to anyone is expressly forbidden. Terms \& 

and-conditions 


\title{
An inverse geometry problem for a one-dimensional heat equation: advances with complex temperatures
}

\author{
Jean-Claude Jolly $^{\mathrm{a} *}$, Laetitia Perez ${ }^{\mathrm{b}}$ and Laurent Autrique ${ }^{\mathrm{a}}$ \\ ${ }^{a}$ Laboratoire d'Ingénierie des Systèmes Automatisés, EA 4094, Université d'Angers-ISTIA, \\ Angers, France; ${ }^{b}$ Laboratoire de Thermocinétique de Nantes, UMR 6607, Ecole Polytechnique de \\ l'Université de Nantes, Nantes Cedex 3, France
}

(Received 12 July 2013; final version received 13 July 2013)

\begin{abstract}
This paper presents an inverse problem in heat conduction, namely the determination of thicknesses of three materials of known heat capacities and thermal conductivities inside a rod of given length subjected to periodic heat flows from measurements of temperatures at both ends. The unknowns are, therefore, the positions of the two interior frontiers between the three materials. Classically, they can be obtained by minimizing the least-squares, non-linear criterion, between the measured and calculated temperatures. Nevertheless, we show that the global minimum providing the solution is close to three local minima that act as traps for a descent algorithm. After providing theoretical justification of the complex temperature method, a method based in this case on the periodicity of boundary fluxes, we suggest a new criterion allowing the global characterization or not of an a priori local minimizer to be tested. It is a criterion of topological nature based on the identification of a singularity.
\end{abstract}

Keywords: heat equation; inverse geometry problem; complex temperatures; identification; global optimization

AMS Subject Classifications: 49K15; 49M30; 49M05

\section{Introduction}

A composite rod made of three materials is considered. This can also be a three-layer plate, modelled in one dimension due to its symmetry. The question that arises is how to determine thicknesses $b_{1}, b_{2}, b_{3}$, given that the total thickness is $b$; in other words, how to determine the position of the two interior border points. For this, we chose a non-invasive thermal process.

Both ends of the rod are subjected to heat flows. These are chosen to be periodic with frequency $\omega$, obtained, for example, from laser sources. We make the assumption that the thermal model is linear, this being realistic for a controlled experiment. We also assume a state of thermal continuity without any thermal contact resistance. These assumptions allow the method of complex temperatures to be applied in order to eliminate time factors in the equations $[1,2]$. Some temperature measurements are performed with frequency $\omega$ at

*Corresponding author. Email: jean-claude.jolly@univ-angers.fr 
both ends. Depending on the real or complex point of view, this defines a non-linear inverse problem for two or four equations and two unknowns.

The problem investigated is one of inverse geometry. This type of problem, often ill-posed, consists of identifying a sub-domain - and its eventual physical characteristics - starting from a direct problem of elliptic or parabolic type in general. This is the case for the direct problem of heat conduction that interests us: it is parabolic in general, but elliptic when stationary. Concerning theoretical aspects, we can cite, for example, the inequalities of global Carleman-type estimations for stability, [3,4] derivation techniques borrowed from shape optimization [5,6] and regularization techniques [7]. Concerning numerical aspects, we can cite, without wanting to be exhaustive, the Galerking methods with mesh generation (FEM),[8] the method of representation with radial basis functions (RBFs),[9] the method of fundamental solutions (MFS),[10] the boundary element method (BEM),[11] the use of genetic algorithms,[12] etc. A few applications are, for example, obstacle problems,[13] the determination of corrosion zones,[14] Spephan phase-change problems,[15] etc.

In comparison to the previous works which are mostly in multi-dimensional domains, the direct and inverse problems that we have defined for the one-dimensional domain are simpler. The periodic flux-type excitation at the rod ends allows us to deal with a direct, elliptic-type problem, but with complex temperatures. This problem has an explicit solution and alternative, stable numerical methods of resolution. As far as the inverse problem of two unknown frontiers is concerned, and, therefore, of finite dimension only two, questions of existence, unicity and stability, without being trivial, are in practice positively solved. To find the solution, a classical least-squares method is used and applied to complex temperature criterion $J$. More precisely, $J$ is modulus quadratic deviation between measured complex temperatures and calculated complex temperatures. This deviation is a non-linear function of the unknown positions of the two interior frontiers. It is a minimization with constraints on the frontiers order. It is at this stage where our results are most important. It is here where the general complexity of inverse geometry problems leads us to be pleased with a local minimizer for the criterion as given by a descent algorithm, numerically showing the presence of several local minimizers for $J$. Moreover, their values are nearer to the global minimum which is equal to zero in principle, but not in practice. This is due to inevitable rounding errors and method errors. We then exploit the introduction of complex temperatures to better determine the global character or not for such a minimizer, the ultimate step of complete resolution. This determination is made by introducing the functionals $K, L$ and $M$, providing new optimization criteria. These criteria are topological in nature because they are based on the identification of singularity.

The paper is organized as follows. A standard thermal model of the direct problem is established. It transfers the geometric unknowns $b_{1}, b_{2}, b_{3}$ to the parametric unknowns $s_{1}, s_{2}$ in the coefficients of the differential equations. Theoretical considerations of existence, uniqueness and stability are discussed. A presentation of the resolution obtained by criterion $J$ is given. The issues of global optimization are detailed. A functional $K$ and the two discontinuity lines of its representative surface are studied. The point at which these lines cross is a singularity point for $K$. An identification criterion $L$ of this $K$ singularity is introduced and compared to $J$. This is followed by the presentation and evaluation of a globality criterion $M$ of an 'all or nothing' type. Some remarks and perspectives are presented in the conclusion. 


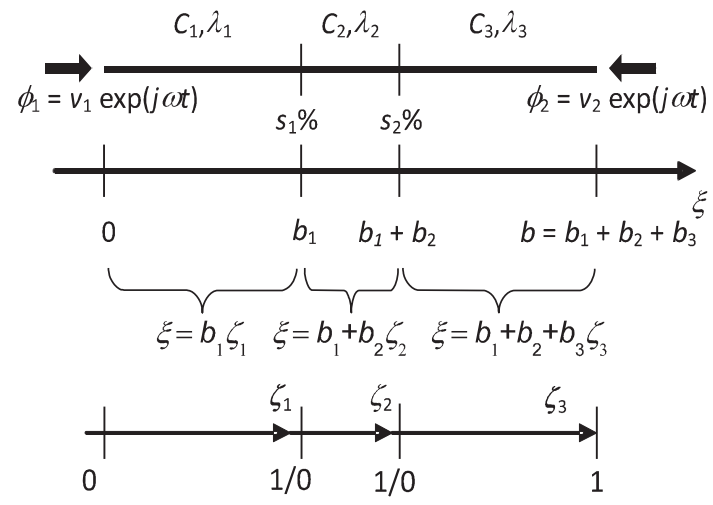

Figure 1. Composite rod.

\section{Thermal model}

The rod (or plate) of fixed length (or thickness) $b$ consists of a stack of three materials Figure 1. The position along the rod is given by a coordinate $\xi \in[0, b]$. The experimental method used is that of complex temperatures.[1,2] This requires, for example, at the left end $\xi=0$ of the rod, two successive sinusoidal heat fluxes with equal magnitudes and with the same temporal frequency $\omega$, but phase-shifted by $\frac{\pi}{2}$, that is to say $v_{1} \cos (\omega t)$ and $v_{1} \sin (\omega t)$. The combination of these two fluxes in $\mathbb{C}$ gives the periodic and complex heat flux

$$
\phi_{1}(t)=v_{1} \cos (\omega t)+j v_{1} \sin (\omega t)=v_{1} \exp (j \omega t)
$$

where $j^{2}=-1$. In the same way, a heat flux $\phi_{2}(t)=v_{2} \exp (j \omega t)$ with the same frequency $\omega$ is applied on the right end $\xi=b$ of the rod. Thermal continuity without any thermal contact resistance is assumed at interior border points. The linear thermal model gives a temperature response $\theta(\xi, t) \in \mathbb{C}$ solution of

$$
C_{i} \frac{\partial \theta}{\partial t}(\xi, t)-\frac{\partial}{\partial \xi}\left(\lambda_{i} \frac{\partial \theta}{\partial \xi}(\xi, t)\right)=0, \quad i=1,2,3, \xi \in[0, b], b=b_{1}+b_{2}+b_{3},
$$

where $C_{i}, \lambda_{i}>0$ are the volumic heat capacities and the conductivities, plus the following relations:

boundary and transition conditions (BC/TC):

$$
\left\{\begin{array}{l}
h_{1} \theta(0, t)-\lambda_{1} \frac{\partial \theta}{\partial \xi}(0, t)=\phi_{1}(t), \quad \phi_{1}(t)=v_{1} \exp (j \omega t) \\
\theta\left(b_{1}-, t\right)=\theta\left(b_{1}+, t\right), \quad \lambda_{1} \frac{\partial \theta}{\partial \xi}\left(b_{1}-, t\right)=\lambda_{2} \frac{\partial \theta}{\partial \xi}\left(b_{1}+, t\right) \\
\theta\left(b_{1}+b_{2}-, t\right)=\theta\left(b_{1}+b_{2}+, t\right), \quad \lambda_{2} \frac{\partial \theta}{\partial \xi}\left(b_{1}+b_{2}-, t\right)=\lambda_{3} \frac{\partial \theta}{\partial \xi}\left(b_{1}+b_{2}+, t\right) \\
h_{2} \theta(b, t)+\lambda_{3} \frac{\partial \theta}{\partial \xi}(b, t)=\phi_{2}(t), \quad \phi_{2}(t)=v_{2} \exp (j \omega t) .
\end{array}\right.
$$

where $h_{1}, h_{2}>0$ are given heat transfer coefficients. 
Note that we have not given any initial conditions; we will return to this in the next section. Instead of this, we make the following hypothesis: the expression $\exp (-j \omega t) \theta(\xi, t)$ is a function $y$ of $\xi$ only, that is to say

$$
\theta(\xi, t)=y(\xi) \exp (j \omega t)
$$

We call (1), (2) and (3) the direct problem.

The assumption (3) allows the factor $\exp (j \omega t)$ to be eliminated in the equations. Thus, a formulation based on partial differential equations for $\theta$ in the space-time variables $(\xi, t)$ becomes a formulation based on ordinary differential equations for $y$ only in the space variable $\xi$. Thereby, in detail, we make the change of variables:

$$
y(\xi)=\left\{\begin{array}{l}
y\left(b_{1} \zeta\right)=x_{1}(\zeta), \quad \text { if } \xi \in\left[0, b_{1}\right] \\
y\left(b_{1}+b_{2} \zeta\right)=x_{2}(\zeta), \quad \text { if } \xi \in\left[b_{1}, b_{1}+b_{2}\right], \quad \text { where } \zeta \in[0,1] . \\
y\left(b_{1}+b_{2}+b_{3} \zeta\right)=x_{3}(\zeta), \quad \text { if } \xi \in\left[b_{1}+b_{2}, b\right] .
\end{array}\right.
$$

Denoting by $c_{i}=j \omega C_{i}, i=1,2,3$, the elimination of factor $\exp (j \omega t)$ in (1) and (2) gives the ODE system

$$
c_{i} b_{i}^{2} x_{i}(\zeta)-\lambda_{i} x_{i}^{\prime \prime}(\zeta)=0, \quad i=1,2,3
$$

subject to

$$
B C / T C:\left\{\begin{array}{l}
b_{1} h_{1} x_{1}(0)-\lambda_{1} x_{1}^{\prime}(0)=b_{1} v_{1} \\
x_{1}(1)=x_{2}(0), \quad b_{2} \lambda_{1} x_{1}^{\prime}(1)=b_{1} \lambda_{2} x_{2}^{\prime}(0) \\
x_{2}(1)=x_{3}(0), \quad b_{3} \lambda_{2} x_{2}^{\prime}(1)=b_{2} \lambda_{3} x_{3}^{\prime}(0) \\
b_{3} h_{2} x_{3}(1)+\lambda_{3} x_{3}^{\prime}(1)=b_{3} v_{2} .
\end{array}\right.
$$

We choose to substitute the three positive variables $b_{1}, b_{2}, b_{3}$ by the two variables $s_{1}, s_{2}$ defined by $b_{1}=s_{1} b, b_{1}+b_{2}=s_{2} b$. Thus, we have

$$
b_{1}=b s_{1}, \quad b_{2}=b\left(s_{2}-s_{1}\right), \quad b_{3}=b\left(1-s_{2}\right) .
$$

Contrary to $b_{1}, b_{2}, b_{3}$ that are linked together by $b_{1}+b_{2}+b_{3}=b$, the variables $s_{1}, s_{2}$ are affinely free, but must satisfy the following constraint given for $s=\left(s_{1}, s_{2}\right)$ :

$$
s \in S=\left\{\left(s_{1}, s_{2}\right) ; 0<s_{1}<s_{2}<1\right\} .
$$

The boundary and transmission conditions become

$$
B C / T C:\left\{\begin{array}{l}
b s_{1} h_{1} x_{1}(0)-\lambda_{1} x_{1}^{\prime}(0)=b s_{1} v_{1} \\
x_{1}(1)=x_{2}(0), \quad b\left(s_{2}-s_{1}\right) \lambda_{1} x_{1}^{\prime}(1)=b s_{1} \lambda_{2} x_{2}^{\prime}(0) \\
x_{2}(1)=x_{3}(0), \quad b\left(1-s_{2}\right) \lambda_{2} x_{2}^{\prime}(1)=b\left(s_{2}-s_{1}\right) \lambda_{3} x_{3}^{\prime}(0) \\
b\left(1-s_{2}\right) h_{2} x_{3}(1)+\lambda_{3} x_{3}^{\prime}(1)=b\left(1-s_{2}\right) v_{2} .
\end{array}\right.
$$

\section{Existence, unicity, and stability}

We review in this section some theoretical issues concerning the direct problem and the inverse problem. 


\subsection{Direct problem}

In connection with the method of complex temperatures, it is useful at this stage to define the solution concept for the direct problem considered. The linear one-dimensional heat equation is perhaps the simplest parabolic partial differential equation. A difficulty arises here with the coefficients (volumic capacity $C$, conductivity $\lambda$ ) that are discontinuous along the rod. Note that the system (1), (2) is given without any initial conditions. Let

$$
\theta(\xi, 0)=\theta_{0}(\xi) \in L^{2}(0, b)
$$

be such a condition. In our case of piecewise constant coefficients, the existence, the uniqueness and the regularity of solutions are given in [16]. But quite curiously, these results, as with more general results on coefficients in $L^{\infty}(0, b),[17,18]$ are widely presented for a uniform volumic heat capacity, i.e. $C_{1}=C_{2}=C_{3}$ constant on $[0, b]$. A simple division by $C_{i}$ in (1) seems to bring us back to this case. This formulation is not correct. When we investigate why, we obtain an inequality which serves to justify the method of complex temperatures.

Let us begin with some notations and definitions. We denote by $C, \lambda$, the piecewise constant functions on $[0, b]$ that take, respectively, the values $C_{1}, \lambda_{1}$ over $\left[0, b_{1}\right), C_{2}, \lambda_{2}$ over $\left(b_{1}, b_{1}+b_{2}\right)$ and $C_{3}, \lambda_{3}$ over $\left(b_{1}+b_{2}, b\right]$.

We define the non-bounded linear operator $M$ by $M u(\xi)=-\frac{d}{d \xi}\left(\lambda(\xi) \frac{d \theta}{d \xi}(\xi)\right)$ with the domain of definition

$$
\begin{aligned}
& D(M)=\left\{u \in H^{1}(0, b) ; \frac{d}{d \xi}\left(\lambda(\xi) \frac{d u}{d \xi}\right) \in L^{2}(0, b),\right. \\
&\left.h_{1} u(0)-\lambda_{1} \frac{d u}{d \xi}(0)=0, h_{2} u(b)+\lambda_{3} \frac{d u}{d \xi}(b)=0\right\} .
\end{aligned}
$$

Note that for an element $u$ in space $D(M)$, this $u(\cdot)$ and $\lambda(\cdot) \frac{d u}{d \xi}(\cdot)$ can be considered continuous [19] and thus some transmission conditions are satisfied, which are precisely:

$$
\left\{\begin{array}{l}
u\left(b_{1}-\right)=u\left(b_{1}+\right), u\left(\left(b_{1}+b_{2}\right)-\right)=u\left(\left(b_{1}+b_{2}\right)+\right) \\
\lambda_{1} \frac{d u}{d \xi}\left(b_{1}-\right)=\lambda_{2} \frac{d u}{d \xi}\left(b_{1}-\right), \lambda_{2} \frac{d u}{d \xi}\left(\left(b_{1}+b_{2}\right)-\right)=\lambda_{3} \frac{d u}{d \xi}\left(\left(b_{1}+b_{2}\right)+\right) .
\end{array}\right.
$$

We define a second non-bounded linear operator $N$ by $N u(\xi)=\frac{1}{C(\xi)} M u(\xi)=$ $-\frac{1}{C(\xi)} \frac{d}{d \xi}\left(\lambda(\xi) \frac{d u}{d \xi}\right)$ with the same domain $D(M)=D(N)$. Equation (1) is an evolution equation that takes the form

$$
\frac{\partial \theta}{\partial t}=-N \theta
$$

Let $(\cdot, \cdot)$ and $|\cdot|$ be the usual Hermitian scalar product and associated norm in $L^{2}(0, b)$. Because $C(\xi) \geq \min \left\{C_{1}, C_{2}, C_{3}\right\}>0$, we have for $L^{2}(0, b)$ the following Hermitian scalar product and associated norm:

$$
\langle u, v\rangle=\int_{0}^{b} C(\xi) u(\xi) \overline{v(\xi)} d \xi=(\sqrt{C} u, \sqrt{C} v),\|u\|=\sqrt{\langle u, v\rangle} .
$$

We denote $E=\left(L^{2}(0, b),\|\cdot\|\right)$. 
We have for $u, v \in D(M)$ :

$$
\begin{aligned}
(M u, v) & =\int_{0}^{b}-\frac{d}{d \xi}\left(\lambda(\xi) \frac{d u}{d \xi}\right) \overline{v(\xi)} d \xi \\
& =\int_{0}^{b} \lambda(\xi) \frac{d u}{d \xi} \frac{\overline{d v}}{d \xi} d \xi+h_{1} u(0) \overline{v(0)}+h_{2} u(b) \overline{v(b)}
\end{aligned}
$$

Since $\lambda$ is bounded over $[0, b]$ with positive bounds, this defines a Hermitian sesquilinear form $e$ over $H^{1}(0, b)$ such that $(M u, v)=e(u, v)$ and which is positive. If $C$ is constant, we can choose to define $e$ with the same properties, but with $N$ instead of $M$ by $(N u, v)=\frac{1}{C}(M u, v)=e(u, v)$. In this case, the operator $N$ is monotone selfadjoint and it is not difficult to verify that it is maximal too. The Hille-Yosida theorem for Hilbert spaces applied to the evolution Equation (10) then gives a solution $\theta$ that satisfies

$$
|\theta(\cdot, t)| \leq \exp \left(-\mu_{0} t\right)\left|\theta_{0}\right|
$$

where $\mu_{0}$ is the smallest eigenvalue of $N$, necessarily non-negative.[20] It is non-zero because the homogeneous boundary conditions in (9) are not of Neumann type for non-zero $h_{1}, h_{2}$.

It is not clear that this last inequality, which is needed to justify the method of complex temperatures, is still true in the case of a non-constant $C$. We are going to show:

Lemm A 3.1 The evolution problem (10) defined for an initial condition $\theta(\xi, 0)=\theta_{0}(\xi) \in$ $D(N)$ has a unique solution $\theta$ with regularity

$$
\theta \in C^{1}([0, \infty) ; E) \cap C([0, \infty) ; D(N)) .
$$

The operator $-N$ is the infinitesimal generator of a $C^{0}$-semigroup of contractions $(T(t))_{t \geq 0}$ defined on $E$. There exists a constant $\mu_{0}>0$ such that

$$
\|T(t)\|_{\mathcal{L}(E)} \leq \exp \left(-\mu_{0} t\right) .
$$

Note that the inequality in Lemma 3.1 is false in general for the norm $|\cdot|_{\mathcal{L}\left(L^{2}(0, b)\right)}$ instead of the norm $\|\cdot\|_{\mathcal{L}(E)}$.

Proof We want to apply the Hille-Yosida theorem for Banach spaces. Let us show that $N$ is a m-accretive operator. The domain $D(N)=D(M)$ is dense in $E$. Let $\mu>0$. We must verify that $I+\mu N$ is bijective from $D(N)$ onto $E$ and that $\left\|(I+\mu N)^{-1}\right\| \leq 1$. Let $f \in E$. We look for $u \in D(N)$ such that $u+\frac{\mu}{C} M u=f$. Because of the homogeneous boundary and transmission conditions satisfied for $u \in D(N)$, an integration by parts leads to

$$
\langle u, v\rangle+\mu e(u, v)=\langle f, v\rangle, \quad \forall v \in H^{1}(0, b)
$$

with

$$
e(u, v)=\int_{0}^{b} \lambda(\xi) \frac{d u}{d \xi} \frac{\overline{d v}}{d \xi} d \xi+h_{1} u(0) \overline{v(0)}+h_{2} u(b) \overline{v(b)}
$$


The sesquilinear form $\langle\cdot, \cdot\rangle+\mu e(\cdot, \cdot)$ is a Hermitian scalar product in $H^{1}(0, b)$. As $\langle f, \cdot\rangle \in$ $H^{1}(0, b)^{\prime}$, the Riesz-Fréchet theorem gives a unique $u \in H^{1}(0, b)$ that verifies (11). We have

$$
\mu \int_{0}^{b} \lambda \frac{d u}{d \xi} \frac{d v}{d \xi} d \xi=\langle f-u, v\rangle, \quad \forall v \in C_{c}^{1}(0, b)
$$

where $C_{c}^{1}(0, b)$ is the space of continuously differentiable functions over $(0, b)$ with compact supports. That shows $\lambda \frac{d u}{d \xi} \in H^{1}(0, b)$. A reversed integration by parts in (11) then shows that $u$ satisfies the homogeneous boundary conditions

$$
h_{1} u(0)-\lambda_{1} \frac{d u}{d \xi}(0)=0, \quad h_{2} u(b)+\lambda_{3} \frac{d u}{d \xi}(b)=0
$$

and thus $u \in D(N)$. So, $I+\mu N$ is bijective from $D(N)$ onto $E$. As $e$ is a positive sesquilinear form, the relation $\|u\|^{2}+\mu e(u, u)=\langle f, u\rangle$ implies $\|u\|^{2} \leq\|f\|\|u\|$ and thus $\left\|(I+\mu N)^{-1}\right\| \leq 1$. Operator $N$ is $\mathrm{m}$-accretive. The Hille-Yosida theorem for Banach spaces gives the conclusion [19,21]. The constant $\mu_{0}$ is the lower bound of all real parts of eigenvalues of $N$ [20]. We will see in the next proposition that $\mu_{0}>0$.

To apply this lemma to the complex temperature method, we also have to consider the evolution problem with non-homogeneous boundary conditions. We summarize some known results in the following proposition, see [18,22] and [23,p.158] (that needs to be adapted for a non-constant $C$ !).

Let us recall that the causal fundamental solution $G\left(\xi, t ; \xi^{\prime}, t^{\prime}\right)$ associated to problem (1), (2) and (8) is the solution of the problem obtained from the original one by adding a term source $\delta\left(\xi-\xi^{\prime}\right) \delta\left(t-t^{\prime}\right)$ and changing the boundary conditions to get homogeneous ones. Moreover, it is required that $G$ is zero for $t<t^{\prime}$.

Proposition 3.2 The problem (1), (2) and (8) has an unique solution $\theta(\xi, t)$ defined by the inverse Fourier transform. The Sturm-Liouville problem which is associated to it has the form

$$
-\frac{d}{d \xi}\left(\lambda(\xi) \frac{d u}{d \xi}(\xi, t)\right)=\mu C(\xi) u(\xi) .
$$

Eigenvalues $\mu$ are positive with the smallest $\mu_{0}>0$. The solution $\theta(\xi, t)$ of the original problem admits the decomposition

$$
\theta=\theta^{0}+\theta^{1}+\theta^{2}
$$

with

$$
\left\{\begin{array}{l}
\theta^{0}(\xi, t)=\int_{0}^{b} C\left(\xi^{\prime}\right) G\left(\xi, t ; \xi^{\prime}, 0\right) \theta_{0}\left(\xi^{\prime}\right) d \xi^{\prime} \\
\theta^{1}(\xi, t)=\int_{0}^{t} G\left(\xi, t ; 0, t^{\prime}\right) \phi_{1}\left(t^{\prime}\right) d t^{\prime} \\
\theta^{2}(\xi, t)=\int_{0}^{t} G\left(\xi, t ; b, t^{\prime}\right) \phi_{2}\left(t^{\prime}\right) d t^{\prime}
\end{array}\right.
$$

where $G$ is the causal fundamental solution. 
The method of complex temperatures is based on the following result:

Theorem 3.3 Let $\theta^{\infty}(\xi, t)=\exp (j \omega t) y(\xi)$ be the solution of (1), (2) with hypothesis (3). It is defined as the unique solution for the initial condition $\theta^{\infty}(\xi, 0)=y(\xi)$. Let $\theta(\xi, t)$ be the solution of (1), (2) for the initial condition (8). Then, $\theta^{\infty}(\xi, t)$ represents the periodic steady part of $\theta(\xi, t)$ in the following sense: in $L^{2}(E)$ we have

$$
\lim _{t \rightarrow \infty} \theta(\cdot, t)=\lim _{t \rightarrow \infty} \theta^{\infty}(\cdot, t) .
$$

Moreover, the following decomposition holds:

$$
\theta=\theta^{\infty}+\theta^{0}, \theta^{0}(\xi, t)=\int_{0}^{b} C(\xi) G\left(\xi, t ; \xi^{\prime}, 0\right)\left(\theta_{0}\left(\xi^{\prime}\right)-y\left(\xi^{\prime}\right)\right) d \xi^{\prime}, \theta^{0}(\cdot, t) \in V,
$$

where $\theta^{0}$ is the solution of problem (1), with (2) transformed to be homogeneous (i.e. $v_{1}=0, v_{2}=0$ ) and with $\theta_{0}-y$ as the initial condition.

Proof Because of the linearity, we have $\psi(\xi, t)=\theta(\cdot, t)-\theta^{\infty}(\cdot, t) \in V$ and $\psi(\cdot, 0)=$ $\theta_{0}\left(\xi^{\prime}\right)-y(\xi)$. We observe that $\psi$ is a solution of the evolution problem (10). Lemma 3.1 gives $\psi=G(t)\left(\theta_{0}-y\right) \in L^{2}(E)$ that satisfies $\|\psi(\cdot, t)\| \leq \exp \left(-\mu_{0} t\right)\left(\theta_{0}-y\right)$. This yields $\lim _{t \rightarrow \infty} \psi(\cdot, t)=0$ in $L^{2}(E)$ and in $L^{2}(0, b)$ too; we get (12). Proposition 3.2 applied to the solution $\psi$ of the problem (1), with (2) as requested, i.e. $v_{1}=0, v_{2}=0$, and with the initial condition $\theta_{0}-y$, gives the expression (13) for $\psi=\theta^{0}$.

\subsection{Inverse problem}

The inverse problem we are interested in is the determination of $s=\left(s_{1}, s_{2}\right)=\left(\frac{b_{1}}{b}, 1-\frac{b_{3}}{b}\right)$ knowing the complex temperatures $X_{1}=x_{1}(0)$ and $X_{2}=x_{3}(1)$. This determination is subject to the constraint (6). Let us denote $X=\left(x_{1}(0), x_{3}(1)\right)$ and $X=A(s)$, where $A$ is an operator from $S$ into $\mathbb{C}^{2}$. The direct problem being well posed, operator $A$ is well defined. We have to study the non-linear inverse operator $A^{-1}$.

If the system (4), (7) and the hypothesis (3) correctly model the phenomenon of heat conduction for the composite rod in a periodic steady state, then this implies the existence of a solution to the inverse problem. Note that in practice the complex measurements $x_{1}(0), x_{3}(1)$ are obtained by measuring $\frac{1}{2 j \omega} \int_{0}^{\frac{\pi}{\omega}} \theta^{\infty}(0, t) d t, \frac{1}{2 j \omega} \int_{0}^{\frac{\pi}{\omega}} \theta^{\infty}(b, t) d t$ or, other averaging summations over a much longer time. This measurement redundancy can mitigate the effect of any disturbance with zero mean.

The unicity can be locally studied from the explicit solution of the direct problem which is possibly deduced from [24], chapter XII, part II, par. 3. According to this author, under the condition of non-nullity of a particular determinant, it is possible to transform the scalar two-point boundary problem (4) and (7) into a matrix Cauchy problem. The verification of the validity conditions of the implicit function theorem for the used simulation values then gives the uniqueness of the solution of the inverse problem. The operator $A$ decomposes into $A=P B$, where $B$ is the operator from $S$ into $C^{\infty}([0, b], \mathbb{C})^{3}$ that, for $s$ associates $\left(x_{1}, x_{2}, x_{3}\right)$ which is the solution of (4) and (7), and where $P$ is the projection of $C^{\infty}([0, b], \mathbb{C})^{3}$ into $\mathbb{C}^{2}$ that for $\left(x_{1}, x_{2}, x_{3}\right)$ associates $\left(X_{1}, X_{2}\right)$. The solution $x_{i}(\xi ; s)$ of (4) and (7) depends on the parameter $s$ with regularity $C^{\infty}$ with respect to $s$, [24]. The 
operator $B$ is, therefore, $C^{\infty}$ on $S$. Its range is a real submanifold $U$ of $C^{\infty}([0, b], \mathbb{C})^{3}$ having dimension two and class $C^{\infty}$. It follows that $A$ is defined from $S$ onto a real submanifold $T$ of $\mathbb{C}^{2}$ having dimension two and class $C^{\infty}$. It is observed that

$$
X_{1}=x_{1}(0), \quad X_{2}=x_{3}(1)
$$

represents four real equations for two real unknowns $s_{1}$ and $s_{2}$. The approach then consists of extracting two real equations of (14) for the application of the inverse function theorem in the neighbourhood of fixed $s_{1}, s_{2}$, as, for example, real part projections, and to consider the two remaining equations as compatibility conditions, imaginary part projections in this case. This must be done under the additional constraint (6). The same inverse function theorem gives regularity $C^{\infty}$ for $A^{-1}$ that is thus locally defined from a neighbourhood of $\left(X_{1}, X_{2}\right) \in T$ into a neighbourhood of $\left(s_{1}, s_{2}\right) \in S$.

The previous approach gives rise to a local unicity result. It is strongly constrained by $S$ and $T$. It can be applied to a candidate solution $\left(s_{1}, s_{2}\right)$ obtained by a numerical method. Because it is difficult to determine the corresponding neighbourhoods, its interest is more theoretical than practical. The existence and uniqueness being acquired in practice, our goal is rather to find a judicious exploitation of the system redundancy (14) to improve the determination of $s$.

The expression $\left(X_{1}, X_{2}\right)$ that could be deduced from [24] would indicate that the edge $\partial S$ is a place of singularity. Numerical instabilities for both the direct and the inverse problems are, therefore, expected at the edge $\partial S$. A second source of instability comes from the constraint $\left(x_{1}, x_{2}, x_{3}\right) \in U$. Indeed, the experimental disturbances and the approximations with which the periodic steady state is observed lead to temperature measurements $\theta(\xi, t)$ that are not made in $\exp (j \omega[0, \infty)) U$ but, let us say, in $C\left([0, \infty), L^{2}(0,1 ; \mathbb{C})^{3}\right)$. But $B$, seen as a compact operator from $S$ into $L^{2}(0,1 ; \mathbb{C})^{3}$, has no bounded inverse. That makes the inverse problem unstable. We can note, however, that for simulations performed in this study, the main noise source comes from the numerical approximations. These can be adjusted at the price of more computation time.

The authors of [4] give a quite general stability result for a composite material in $\mathbb{R}^{n}$ obtained (for a constant $C$ !) with the help of Carleman-type estimates. It can be applied to our inverse-geometry problem, but with flux measurements at the boundary instead of temperature measurements. This is not essential for us since all techniques developed here apply. Let $T>0$ be a terminal time and let $a=\frac{\lambda}{C}$ be the diffusivity, which is a piecewise constant for us and that includes unknown interior border points. Their result concerns the operator that maps the diffusivity $a$ and the initial conditions $\theta_{0}$ to the gradient at the boundary $\left.\frac{\partial \theta}{\partial n}\right|_{0, b}$ and the Laplacien in the $\left.\operatorname{rod} \frac{\partial^{2} \theta(\cdot, T)}{\partial \xi \partial \xi}\right|_{[0, b]}$. They show that the operator is injective and that there is linear stability for the diffusivity and logarithmic stability for the initial conditions. The used second-order derivative on the whole rod to control the diffusivity variation is limiting for us. As we are only interested in the steady periodic state, is this necessary? We will not expand on this issue.

Let us also mention that the conjugate gradient algorithm is known for its regularizing properties [23]; this will be used in Section 4.3.

We illustrate the stability behaviour of the inverse problem in our simulations by adopting a measurement error $\Delta X$ equal to $(0.01 \mathrm{~K})$, which is realistic for a temperature measurement in the laboratory. In Figures $2-8$, the associated solution is $s^{* *}$, whereas the exact solution is $s^{*}$. 


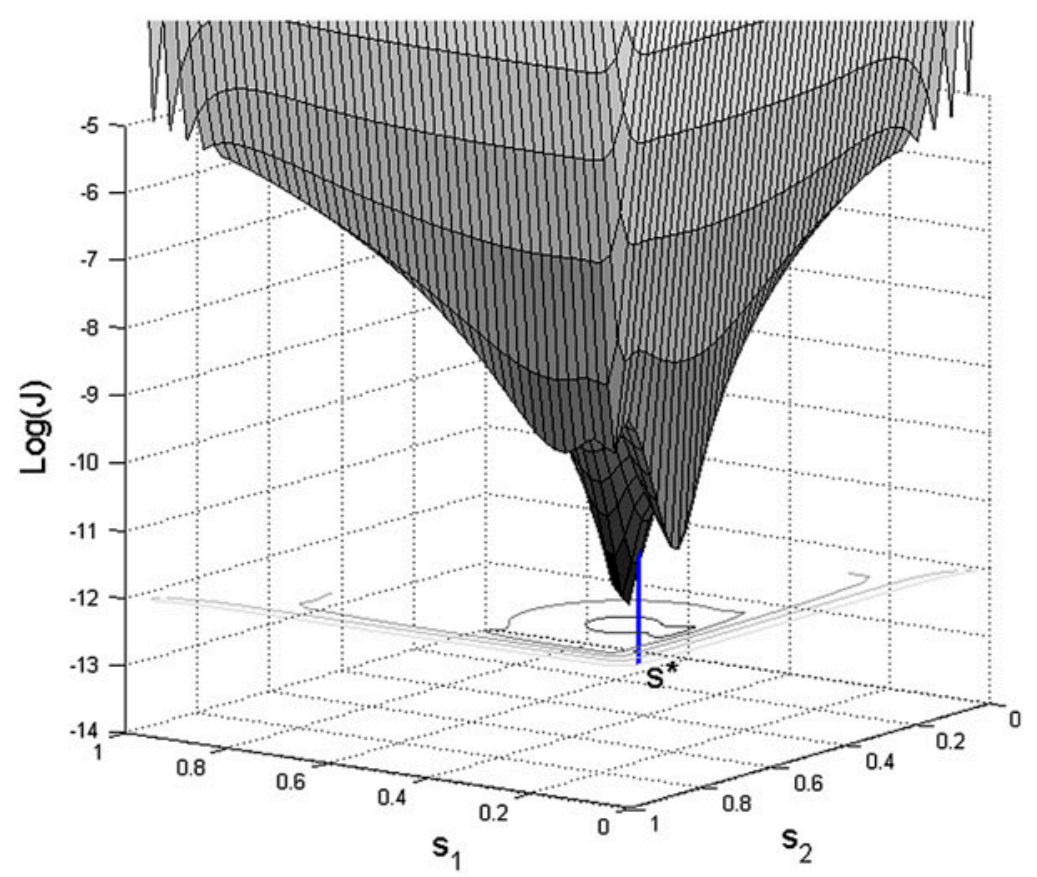

Figure 2. Log of the modulus criterion $J$.

\section{Direct problem}

\subsection{Resolution}

System (4) and (7) is a two-point boundary value problem together with some transmission conditions. It cannot be solved by current versions of Maple@ $\odot$ or Matlab@. Following [24], chapter XII, part II, par. 3, it can be shown that there exists a unique solution with an explicit formula. For lack of place, we do not give it here.

Prior to this study, a numerical scheme based on a Haar wavelet devoted to (4) and (7) has been developed and tested.[25] A third method of finite element type consists of solving directly the PDE (1), (2) and (8) rather than the ODE system (4) and (7). For this, it is necessary to work with a solver that accepts the transition conditions, as is the case of Comsol@.

We have tested the three methods; they give consistent results. We chose to use the Haar wavelet scheme; it seems to lead to more stable computations (with only additions and multiplications) than with the explicit formula (combination of exponentials with low and high arguments). For the FEM, some limitations appear for transition conditions that will not be discussed here.

\subsection{Data}

For the inverse problem considered here, measurements $X^{*}=\left(X_{1}^{*}, X_{2}^{*}\right)$ correspond to the desired geometric solution

$$
s^{*}=(0.2,0.7)
$$




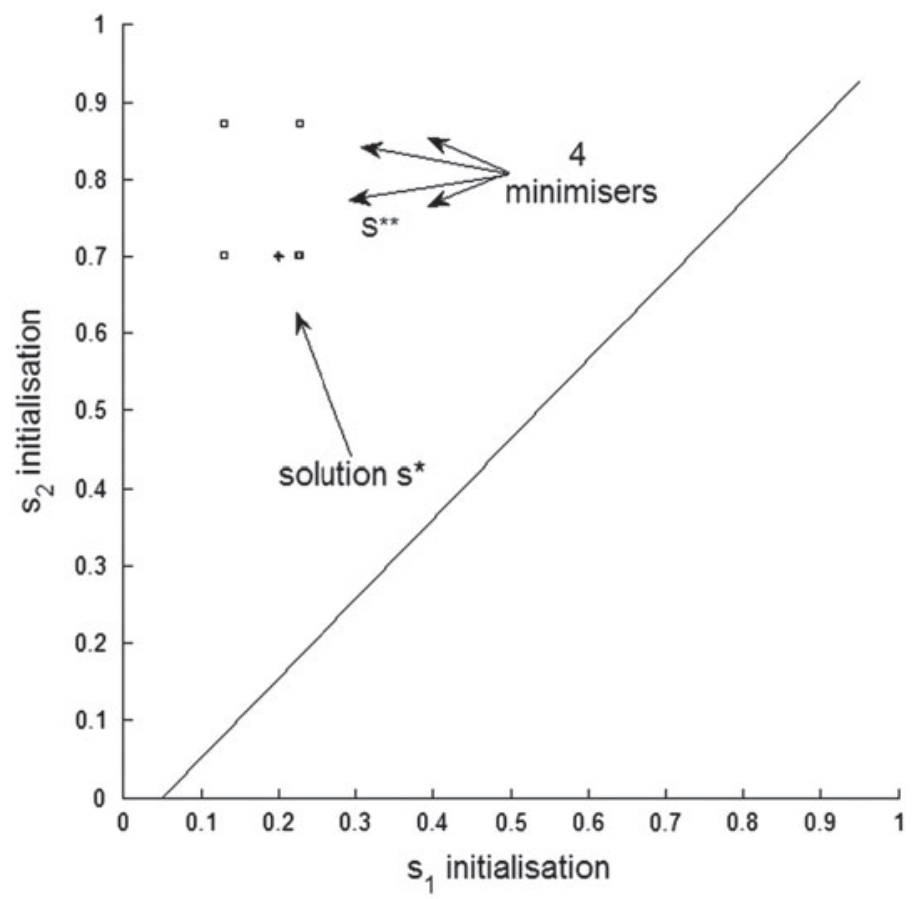

Figure 3. Four minimizer candidates obtained for 1326 uniformly distributed initialization points. Computation time: $11253 \mathrm{~s}$.

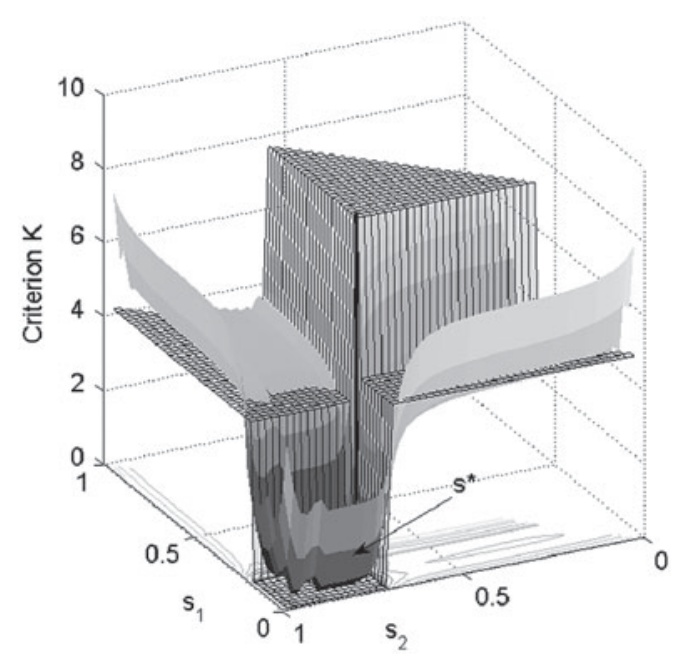

Figure 4. Argument criterion $\mathrm{K}$ with its singularity $\mathrm{K}_{0}(\theta)$ (grid with transparency). 


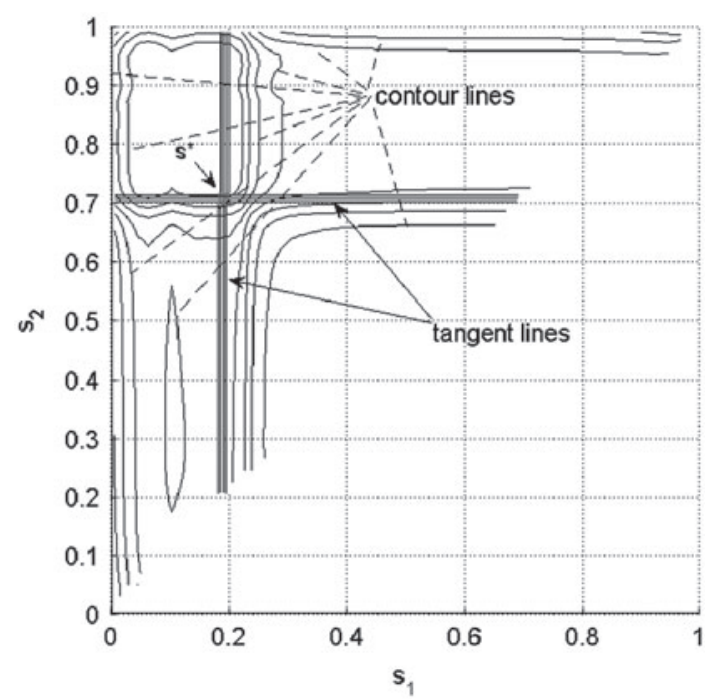

Figure 5. Argumnet criteria K: tangent lines to the singularity.

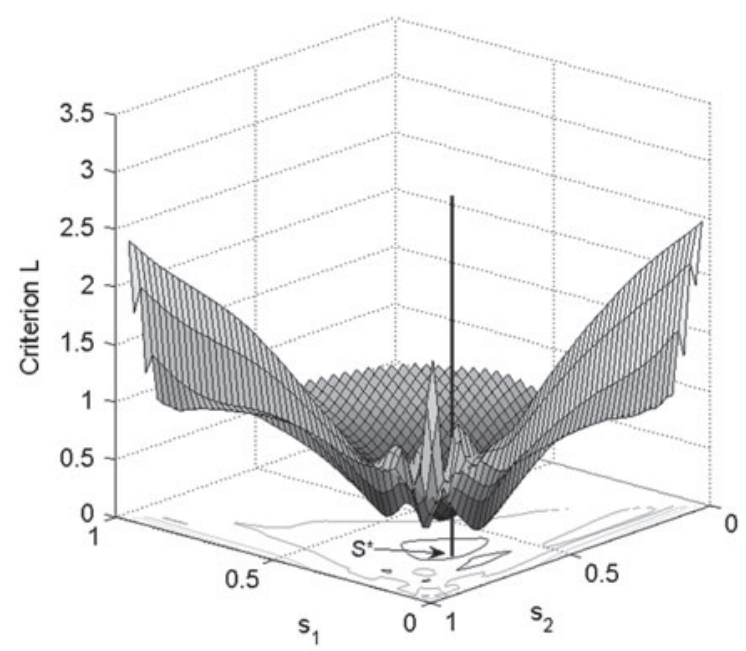

Figure 6. Image criterion $\mathrm{L}$ with a global minimum.

disturbed by a truncation $\Delta X$ to a hundredth of a degree. Thus, we do not have $X^{*}=A\left(s^{*}\right)$, but rather $X^{*}=A\left(s^{* *}\right)$. In this way, a measurement error $\Delta X$ is included in $X^{*}=$ $A\left(s^{*}\right)+\Delta \mathrm{X}$ which is of the order $0.01 \mathrm{~K}$. Of course, if the algorithms converge, then they will converge to $s^{* *}$ instead of $s^{*}$. The simulations are performed for the following numerical values: 


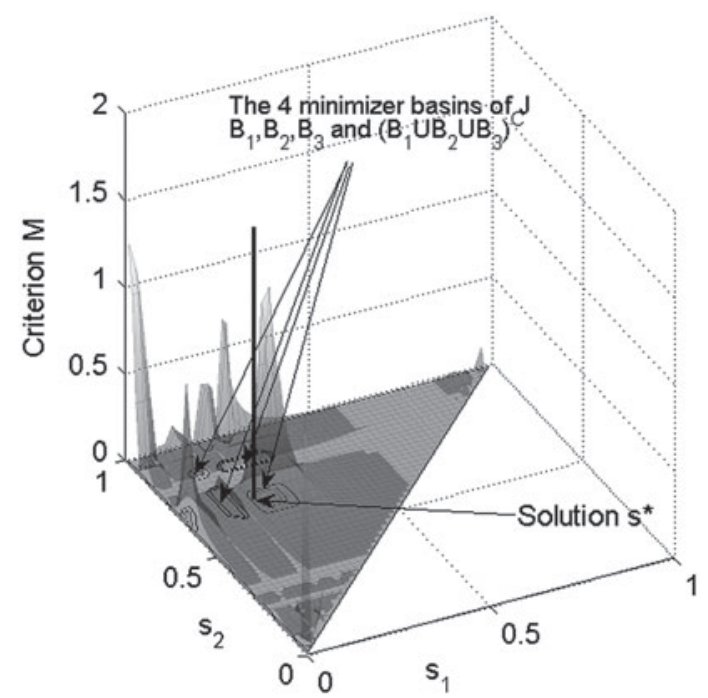

Figure 7. Min-grad citerion M (by transparency).

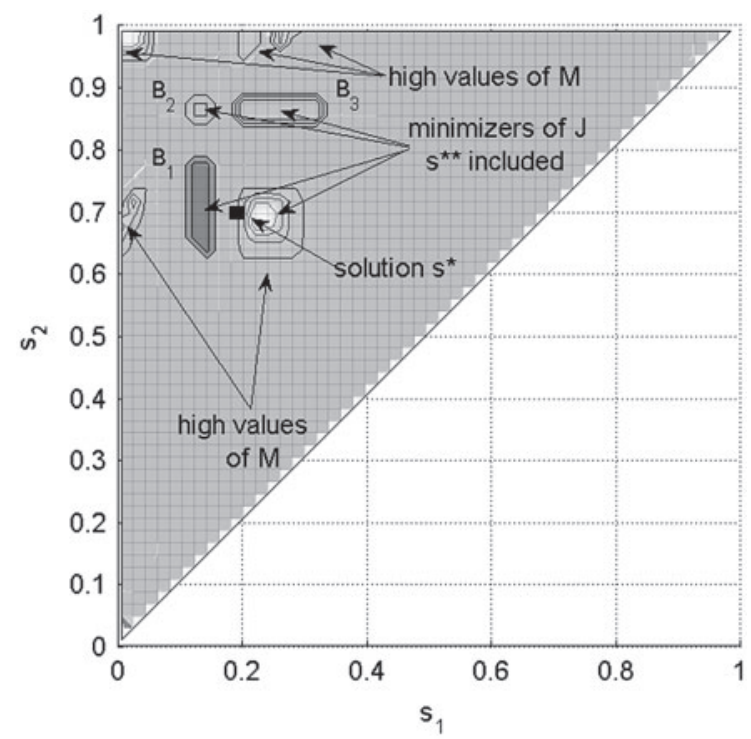

Figure 8. The solution given by max $\mathrm{M}$ and $\min \mathrm{J}$.

- Materials data:

$$
\left\{\begin{array}{l}
\lambda_{1}=\lambda_{3}=1.4 \mathrm{~W} / \mathrm{m} / \mathrm{K}, \quad C_{1}=C_{3}=1.5 \times 10^{6} \mathrm{~J} / \mathrm{m}^{3} / \mathrm{K}, \\
\lambda_{2}=21.9 \mathrm{~W} / \mathrm{m} / \mathrm{K}, \quad C_{2}=2.3 \times 10^{6} \mathrm{~J} / \mathrm{m}^{3} / \mathrm{K}, \quad b=5 \times 10^{-3} \mathrm{~m},
\end{array}\right.
$$

which is a titanium plate clamped between two glass plates. 
- Experimental data:

$$
h_{1}=h_{2}=20 \mathrm{~W} / \mathrm{m}^{2} / \mathrm{K}, \quad v_{0}=10^{4} \mathrm{~W} / \mathrm{m}^{2}, \quad \omega=30 \mathrm{~s}^{-1},
$$

and $\left(v_{1}, v_{2}\right)=\left(v_{0}, v_{0}\right)$ which corresponds to the same heat fluxes and to the same exchange coefficients with ambient air at the boundaries.

\subsection{First-order derivatives}

The determination of partial derivatives $\frac{\partial x_{1}(0)}{\partial s_{k}}, \frac{\partial x_{3}(1)}{\partial s_{k}}, k=1,2$, is necessary for the gradient computation of some criteria, see Section 5. It is also needed for the computation of some tangent lines, see Section 8 .

The easiest way to proceed is to solve the sensitivity problem. However, it is possible to get the first-order derivatives by defining an adjoint problem [25]. It has several advantages, but the formulae are more complicated.

Consider $b_{1}, b_{2}, b_{3}$ as free variables. Write (4) and (7) for $b_{j}+\delta b_{j}$ instead of $b_{j}$; its solution is $\left(x_{i}+d x_{i}\right)\left(\zeta_{i}\right)$. Subtract (4) and (7) and discard second-order terms and higher order ones. This yields

$$
\begin{gathered}
b_{i}^{2} c_{i} d x_{i}\left(\zeta_{i}\right)-\lambda_{i} d x_{i}^{\prime \prime}\left(\zeta_{i}\right)=-2 b_{i} \delta b_{i} c_{i} x_{i}\left(\zeta_{i}\right), \quad i=1,2,3 \\
B C / T C: \\
\left\{\begin{array}{l}
b s_{1} h_{1} d x_{1}(0)-\lambda_{1} d x_{1}^{\prime}(0)=\left(v_{1}-h_{1} x_{1}(0)\right) \delta b_{1}, \\
d x_{1}(1)=d x_{2}(0), \\
\lambda_{1} x_{1}^{\prime}(1) \delta b_{2}+b\left(s_{2}-s_{1}\right) \lambda_{1} d x_{1}^{\prime}(1)=\lambda_{2} x_{2}^{\prime}(0) \delta b_{1}+b s_{1} \lambda_{2} d x_{2}^{\prime}(0), \\
d x_{2}(1)=d x_{3}(0), \\
\lambda_{2} x_{2}^{\prime}(1) \delta b_{3}+b\left(1-s_{2}\right) \lambda_{2} d x_{2}^{\prime}(1)=\lambda_{3} x_{3}^{\prime}(0) \delta b_{2}+b\left(s_{2}-s_{1}\right) \lambda_{3} d x_{3}^{\prime}(0), \\
\alpha_{3,2} d x_{3}(1)+\lambda_{3} d x_{3}^{\prime}(1)=\left(v_{2}-h_{2} x_{3}(1)\right) \delta b_{3} .
\end{array}\right.
\end{gathered}
$$

Note that all this is justified since the solution $x_{i}(\zeta ; s)$ of (4) and (7) is continuously differentiable with respect to $s$.[24]

Remembering (5), we find that the variation $\delta s=\left(\delta s_{1}, \delta s_{2}\right)$ is related to $\delta b_{i}$ by

$$
\delta b_{1}=b \delta s_{1}, \quad \delta b_{2}=b\left(\delta s_{2}-\delta s_{1}\right), \quad \delta b_{3}=-b \delta s_{2} .
$$

We also have

$$
d x_{1}(0)=\frac{\partial x_{1}(0)}{\partial s_{1}} \delta s_{1}+\frac{\partial x_{1}(0)}{\partial s_{2}} \delta s_{2}, \quad d x_{3}(1)=\frac{\partial x_{3}(1)}{\partial s_{1}} \delta s_{1}+\frac{\partial x_{3}(1)}{\partial s_{2}} \delta s_{2} .
$$

Proposition 4.1 Let $x_{i}\left(\zeta_{i}\right)$ be the solution of the direct system (4) and (7). Consider the resolution of the sensitivity system $(15)$ and $(16)$. The choice $\delta s=(1,0)$ gives a solution that is $\frac{\partial x_{i}(\zeta)}{\partial s_{1}}$ and the choice $\delta s=(0,1)$ gives a solution that is $\frac{\partial x_{i}(\zeta)}{\partial s_{2}}$. The first-order derivatives $\frac{\partial x_{1}(0)}{\partial s_{k}}, \frac{\partial x_{3}(1)}{\partial s_{k}}, k=1,2$ follow from evaluations at $\zeta=0,1$. 


\section{Least-squares criterion in modulus $J$}

We want to solve $X=A(s), s \in S$. We distinguish between the searched solution and the associated boundary temperatures, that is to say, the measured temperatures, by the notations $s^{*}$ and $X^{*}$. Recall that we have to take into account an error measurement that makes $s^{*}$ approximated by $s^{* *}$ such that $X^{*}=A\left(s^{* *}\right)$. A first idea consists of solving $\left|X_{1}-X_{1}^{*}\right|=0,\left|X_{2}-X_{2}^{*}\right|=0$ with a Newton method. Other choices are possible as, for example, $X_{1}-X_{1}^{*}=0$ taking $X_{2}-X_{2}^{*}=0$ as a constraint. In all cases, this algorithm is badly adapted to take into account the different constraints, at least $s \in S$. For this reason, we prefer an optimization method under constraints using standard software. We chose the function fmincon of the Matlab@R2012a optimization toolbox with the interior point algorithm option. It is better suited when the true gradient of the criterion can be provided, this being the case for us. As the conjugate gradient algorithm is used in some steps of fmincon, we can expect regularising behaviour of this algorithm.

A natural criterion is one of the least squares in modulus:

$$
J(s)=\frac{1}{2}\left(\left|X_{1}-X_{1}^{*}\right|^{2}+\left|X_{2}-X_{2}^{*}\right|^{2}\right) .
$$

We call it the modulus criterion. Let us give its gradient. We rewrite (17) as $J(s)=$ $\frac{1}{2}\left|X-X^{*}\right|^{2}$ with $X=A(s)$ in $\mathbb{C}$. For any $\delta s \in \mathbb{R}^{2}$, we have

$$
\begin{aligned}
J_{s}^{\prime} \cdot \delta s & =\frac{1}{2}\left(\left(A_{s}^{\prime} \cdot \delta s, A(s)-X^{*}\right)+\left(A(s)-X^{*}, A_{s}^{\prime} \cdot \delta s\right)\right) \\
& =\frac{1}{2}\left(\overline{\left(A(s)-X^{*}, A_{s}^{\prime} \cdot \delta s\right)}+\left(A(s)-X^{*}, A_{s}^{\prime} \cdot \delta s\right)\right) \\
& =\operatorname{Re}\left(\left(A(s)-X^{*}, A_{s}^{\prime} \cdot \delta s\right)\right) \\
& =\operatorname{Re}\left(\left(\left(A_{s}^{\prime}\right)^{*} \cdot\left(A(s)-X^{*}\right), \delta s\right)\right)=\left(\operatorname{Re}\left(\left(A_{s}^{\prime}\right)^{*} \cdot\left(A(s)-X^{*}\right)\right), \delta s\right)
\end{aligned}
$$

and so $J_{s}^{\prime}=\operatorname{Re}\left(\left(A_{s}^{\prime}\right)^{*} \cdot\left(A(s)-X^{*}\right)\right)$. Here, $\left(A_{s}^{\prime}\right)^{*}$ is the adjoint operator of

$$
A_{s}^{\prime}=\left(\begin{array}{ll}
\frac{\partial x_{1}(0)}{\partial s_{1}} & \frac{\partial x_{3}(1)}{\partial s_{1}} \\
\frac{\partial x_{1}(0)}{\partial s_{2}} & \frac{\partial x_{3}(1)}{\partial s_{2}}
\end{array}\right)
$$

that has been computed in Section 4.3.

Figure 2 gives the graphical representation of criterion $J$ on $S$ on a logarithmic scale. It suggests the existence and the unicity of a global minimizer, $s^{* *}=(0.2260,0.7022)$ in this case.

Recall that $\left(s_{1}, s_{2}\right)$ is a way to model some kind of piecewise definitions for $C$ and $\lambda$ along the rod. By looking at Figure 2, we can roughly make the assumption that the piecewise constant parameters $C$ and $\lambda$, at sufficient distance from $\partial S$, appear to have logarithmic stability with respect to the boundary temperatures. This is to be compared with [4], already cited.

More interesting is the presence of several local minimizers for $J$ that are quite close to each other, as suggested by Figure 2 . We get a numerical confirmation with the used descent algorithm fmincon by varying the initialization point $s^{0} \in S$ and visualizing all exit 
points. For 1326 points $s^{0}$ uniformly distributed in $S$, we get four local minimizers for $J$ shown in Figure 3. They are marked by four square points, with one of them $s^{* *}$ that is close to the exact solution $s^{*}$ represented by a cross. We are thus dealing with a problem of global optimization for a non-convex criterion. In Figures 7 and 8, we marked three surfaces $B_{1}, B_{2}, B_{3}$ which are oblong, fairly regular and focused on the three local minimizers of $J$ other than $s^{* *}$. These areas represent the basins of attraction of the minimizers for the descent algorithm initialized by $s^{0}$. More specifically, they were obtained as contour lines of the minimum of $J$, as a function of $s^{0}$, according to a colour scale. This explains the smooth appearance of the border lines. Their complementary in $S$ is the basin of attraction associated to the fourth minimizer of $J$, i.e. $s^{* *}$. If we return to the inverse problem posed, the basins $B_{1}, B_{2}, B_{3}$ materialize the risk of error associated to the resolution by $f$ mincon according to the chosen initialization point $s^{0}$. We can notice that in this example, the area $B_{1} \cup B_{2} \cup B_{3}$ is small compared to that of its complement in $S$, about $4 \%$. This means that the aforementioned risk is reduced. Nevertheless, the basins $B_{1}, B_{2}, B_{3}$ are quite close to the estimated solution $s^{* *}=(0.2260,0.7022)$, which can be interpreted as an increased risk factor.

Either visually in Figure 3, or with $f$ mincon in Figure 4, we obtain all the local minimizers and, therefore, the solution $s^{* *}$. This resolution is affected by uncertainties in temperature measurements. It also assumes that we have been able to vary sufficiently $s^{0}$ in $S$, for example, by using a grid of a sufficiently small step size $\Delta$, in order not to miss a minimizer. The situation looks favourable for this simulation. Indeed, the basin of the global minimizer is the largest one. If this fact could be established more generally, this would suggest the following rule: that a minimizer as a solution candidate is even more probably the true solution when the descent algorithm gives the same minimizer for different initialization points $s^{0}$ that are far away from each other.

But if the stepsize $\Delta$ is as small as the precision expected for $s^{*}$, then a descent algorithm makes no sense: a simple sorting algorithm gives the global minimizer. That is the case here. Nevertheless, we have in mind to solve inverse problems that lead to a more general $n$ parameter criterion. For example, such a criterion could be the one for the determination of $n=4$ unknown interior border points. The four temperature measurements needed would again be obtained at the two boundaries, but doubled for two different frequencies $\omega_{1}$ and $\omega_{2}$. The sorting algorithm in dimension $n$ is rapidly inefficient since its complexity order is exponential with $n$.

Let us now suppose, as in our application, that a random choice of the initialization point $s^{0}$ for the descent algorithm rapidly gives a minimizer $s^{* 1}$ that has a good chance to be the desired solution. Could we find a criterion that can check if a local minimizer has the property of globality or not? We could believe that the verification of $J=0$ is sufficient to decide if a given minimizer is the global one or not. But, this is not the case because of the unavoidable numerical and measurement approximations that prevent us to decide when the value of $J$ is close to zero or not, see Figure 2. Thus in our application, the three minimizers associated to the basins $B_{1}, B_{2}, B_{3}$ give the respective values for $J: 1.30 \times 10^{-5}, 1.07 \times 10^{-4}, 1.02 \times 10^{-4}$ against $7.60 \times 10^{-6}$ for $s^{* *}$. The choice $s^{0}=(0.12,0.67)$ with $f$ mincon gives the local minimizer $s^{* 1}=(0.1286,0.7021)$ in $B_{1}$, see also Figure 3. A misinterpreted assessment of $J\left(s^{* 1}\right)=1.30 \times 10^{-5}$ could lead to retain $s^{* 1}$ instead of $s^{* *}$.

Keeping in mind an $n$-dimensional inverse problem, $n>2$, we explore below for $n=2$ some new criteria to test if a local minimizer is global or not. 


\section{Least-squares criterion in argument $K$}

Criterion $J$ assesses the modulus of a difference. For complex temperatures, it is quite natural to seek additional information by looking at the argument of a difference. We define the criterion

$$
K(s)=\frac{1}{2}\left(\arg ^{2}\left(X_{1}-X_{1}^{*}\right)+\arg ^{2}\left(X_{2}-X_{2}^{*}\right)\right) .
$$

We call it the argument criterion. The nullity of $K$ provides the equality of the arguments of $X_{1}, X_{1}^{*}$ on one hand and $X_{2}, X_{2}^{*}$ on the other hand. It does not provide the equality of moduli. The relation (18) thus defines a partial optimization criterion. However, we can see that the relation $K$ allows a good determination of the solution $s^{*}$. Indeed, its graphic representation in $\mathbb{R}^{3}$ reveals two lines of strong discontinuity that are substantially straight, and whose intersection is $s^{*}$, or more exactly $s^{* *}$, see Figure 4 . How can this be explained?

Let us set

$$
s_{1}=s_{1}^{*}+r \cos (\theta), \quad s_{2}=s_{2}^{*}+r \sin (\theta), \quad r \geq 0
$$

which is a polar representation of $s$ centred at $s^{*}$ (the choice of $s^{* *}$ would be more accurate). A limited expansion with respect to $r$ and for fixed $\theta$ gives

$$
X_{1}-X_{1}^{*}=r\left(R_{1}(\theta)+O_{r}(1)\right), \quad X_{2}-X_{2}^{*}=r\left(R_{2}(\theta)+O_{r}(1)\right),
$$

where

$$
\begin{gathered}
R_{1}(\theta)=\partial_{1} X_{1}^{*} \cos (\theta)+\partial_{2} X_{1}^{*} \sin (\theta), \quad R_{2}(\theta)=\partial_{1} X_{2}^{*} \cos (\theta)+\partial_{2} X_{2}^{*} \sin (\theta), \\
\partial_{l} X_{k}^{*}=\partial_{l} X_{k}\left(s^{*}\right), \quad k, l=1,2 .
\end{gathered}
$$

We have

$$
\arg \left(X_{1}-X_{1}^{*}\right)=\arg \left(R_{1}(\theta)+O(1)\right), \arg \left(X_{2}-X_{2}^{*}\right)=\arg \left(R_{2}(\theta)+O(1)\right) .
$$

The limit of (18) with respect to $r$ for fixed $\theta$ is

$$
K_{0}(\theta) \triangleq \lim _{s \rightarrow s^{*}, \theta \text { fixed }} K(s)=\frac{1}{2}\left(\arg ^{2}\left(R_{1}(\theta)\right)+\arg ^{2}\left(R_{2}(\theta)\right)\right) .
$$

Criterion $K(s)$ has a singularity at $s^{*}$ (multivaluation $K_{0}(\theta)$ ). See Figure 4 where the graphical representation of the function $(r, \theta) \mapsto K_{0}(\theta)$ has been superimposed over that of $K(s)$. The graphical representation of $K$ is given for a fixed determination of the multivalued function $\arg$, for example, with values in $(-\pi, \pi]$. Therefore, there is a discontinuity when

$$
\operatorname{Im}\left(R_{k}(\theta)\right)=0 \text { and } \operatorname{Re}\left(R_{k}(\theta)\right)<0, \quad k=1 \text { or } k=2 .
$$

A remarkable observation made in Figure 4 and also in Figure 5, its projected view on $S$, is the quality of the approximation of $K(s)$ by $K_{0}(\theta)$, i.e. at order 0 with respect to $r$ :

$$
K(s)=K_{0}(\theta)+O_{r}(1) .
$$

In Section 7, we will assume the validity of this approximation in order to propose an image criterion where the singularity acts like a weighted factor.

Let us denote $T_{1}, T_{2}$ the tangent lines to the discontinuity lines of $K(s)$ in the $s$-plane. Their equations are

$$
a_{k, 1} \sin \left(\alpha_{k, 1}\right)\left(s_{1}-s_{1}^{*}\right)+a_{k, 2} \sin \left(\alpha_{k, 2}\right)\left(s_{2}-s_{2}^{*}\right)=0, \quad k=1 \text { for } T_{1}, k=2 \text { for } T_{2},
$$


where $a_{k, l}, \alpha_{k, 2}$ are defined by $\partial_{l} X_{k}^{*}=a_{k, l} \exp \left(j \alpha_{k, l}\right), k, l=1,2$. The angle $\theta^{*}$ between $T_{1}$ and $T_{2}$ is given by

$$
\tan \left(\theta^{*}\right)=-\frac{a_{k, 1} \sin \left(\alpha_{k, 1}\right)}{a_{k, 2} \sin \left(\alpha_{k, 2}\right)} .
$$

Figure 5 shows the graphical representation of $T_{1}, T_{2}$ together with the contour lines of $K(s)$ in the $s$-plane. In Section 8 , we will assume the validity of the approximation of the discontinuity contour lines of $K(s)$ by $T_{1}, T_{2}$ in order to propose a min-gradient criterion where the tangent lines $T_{1}, T_{2}$ act as strong gradient sensors.

\section{Topological criterion in image $L$}

To simplify the notations as we started to do in the last paragraph, let us now forget, as well as for the following sequel, the distinction between $s^{*}$ and $s^{* *}$.

We try to identify the location of the singularity observed for $K$ (Figure 4). We note that $K$ given by (18) depends on $s^{*}$. Now let us note $K_{*}(s)$ instead of $K(s)$ for $s^{*}$ corresponding to the measurements. Let us denote more generally $K_{s}(\sigma)$ instead of $K(\sigma)$ if $s$ is a variable parameter corresponding to some variable temperature measurements $\widehat{X(s)}$ which are functions of $s$. Quite naturally, we define a criterion for a difference in $L^{1}(S)$, namely:

$$
L(s)=\int_{S_{0}}\left|K_{s}(\sigma)-K_{*}(\sigma)\right| d \sigma=\left\|K_{s}-K_{*}\right\|_{L^{1}\left(S_{0}\right)}
$$

for a fixed $S_{0} \subset S$ such that points too near to $\partial S$ are excluded. Indeed, some singularities appear in $\partial S$. We call it the image criterion. Numerically, a discretization of $S_{0}$ is needed to evaluate the integral. We choose a grid $S_{n} \subset S_{0}$ of $n$ points uniformly distributed with respect to a step size $\Delta$ for either $s_{1}$ or $s_{2}$. Thus, criterion $L$ is approximated by

$$
L_{n}(s)=\Delta^{2} \sum_{\sigma_{i} \in S_{n}}\left|K_{s}\left(\sigma_{i}\right)-K_{*}\left(\sigma_{i}\right)\right|
$$

Let $n=\frac{1}{2} n_{0}\left(n_{0}+1\right)=1326$, where $n_{0}=51$ is the number of discretization points on one side of $S$. The graphic representation of the corresponding criterion $L_{n}$ is given in Figure 6. A remarkable analogy with the modulus criterion $J$ is the presence of the same three local minimizers and of the same global minimizer $s^{*}$. This can be seen by examining the corresponding values.

We are interested in the determination of $s^{*}$ by $L$. The calculation of $L_{n}(s)$ is approximated by that of $L_{n}\left(s_{k}\right)$, where $s_{k}$ is the point of $S_{n}$ closest to $s$. The computation time and the accuracy for a minimization algorithm are related to $n$. The accuracy of $s^{*}$ under these conditions is of order $\frac{1}{n_{0}-1}=0.02$. The calculation of

$$
K_{s}(\sigma)=\frac{1}{2}\left(\arg ^{2}\left(X_{1}(\sigma)-\widehat{X_{1}(s)}\right)+\arg ^{2}\left(X_{2}(\sigma)-\widehat{X_{2}(s)}\right)\right)
$$

that is for $(s, \sigma) \in S_{n}^{2}$ is reduced to $n$ resolutions of (4) and (7) giving $X_{l}(\sigma), \sigma \in S_{n}$, $l=1,2$. We can deduce immediately the $n$ values of $L_{n}\left(S_{n}\right)$. A sorting algorithm of complexity $O\left(n^{2}\right)$ or $O(n \log (n))$ (function min of Matlab) then gives the global minimizer of $L$. The last two steps are of negligible cost in time compared to the calculation of $X_{l}\left(S_{n}\right), l=1,2$. Note that the use of such a sorting algorithm to determine $s^{*}$ applies for $J$, as well as for $L$ with the same number $(n=1326)$ of calls to the criterion. This 
is to be compared with the 60000 calls to $J$ by fmincon to obtain Figure 6. As already mentioned, the cost of this global minimization sorting algorithm varies exponentially with the dimension of the parameter space $S$, that is equal to two here. This preserves the interest of using a descent algorithm and of the study of an alternative method oriented towards global minimization. Three conclusions can be drawn:

- The remarkable similarity in terms of local extrema of $L$ and $J$ confirms the characterization of $s^{*}$ as a topological singularity of $K_{*}$.

- The sorting algorithm, with either $J$ or $L$, is the most effective for a global minimimum search when working in a parameter space $S$ with a low-dimension (two in this case).

- For higher a dimension of space $S$, the descent algorithm is the right tool. Moreover, the definition by summation on $S$ of $L$ disqualifies the use of $L$ in this way.

\section{Topological criterion in min-gradient $M$}

For our application, the function fmincon initialized by a random choice of $s^{0}$ gives a minimizer $s^{* \times}$ of $J$ with 45 calls on average to the criterion. In addition, we observed that there is a good chance that this minimizer is a global one, i.e. $s^{* \times}=s^{*}$, which solves the inverse problem under consideration. Can we confirm or deny the identity $s^{* \times}=s^{*}$ with fewer calls to the criterion (to be detailed) than the 1236 calls of either $J$ or $L$ given by the sorting algorithm of the previous section to obtain $s^{*}$ ? We make a proposal in this direction by introducing a new topological criterion $M$.

Let us consider $T_{s}=T_{1, s} \cup T_{2, s}$, where $T_{1, s}, T_{2, s}$ are the tangent lines to the discontinuity contour lines of $K_{s}(\sigma)$ as defined in (19) (to be read in place of $K_{s^{*}}(s)$ ). Let $K_{*}^{n}(\sigma)$ be a sufficiently regular approximation of $K_{*}(\sigma)$, obtained by using a grid discretization $S_{n}$ of $S$ (see (20)) and a smoothing. Note that $K_{*}(\sigma)$ is issued from the measurements $\hat{X}$ and from the thermal model (4) and (7) for $\sigma$ in place of $s$. We define the topological criterion $M$ as follows:

$$
M_{n}(s)=\min _{\sigma \in T_{s}}\left\|\nabla K_{*}^{n}(\sigma)\right\|_{2}
$$

where $\|\cdot\|_{2}$ is a norm of $\mathbb{C}^{2}$. We call it the min-grad criterion. This is the minimal norm of the gradient $\nabla K_{*}^{n}(\sigma)$ of a point $\sigma$ in $T_{S} \subset S$.

Its consideration is based on the following arguments:

- If $s=s^{*}$, then $K_{*}(\sigma)$ varies greatly (discontinuity) at the point of singularity $s^{*}$, but also all along $T_{s^{*}}$, due to the supposed quality of the approximation of $K_{*}(\sigma)$ by $s=(r, \theta) \mapsto K_{0}(\theta)$. This implies high values for $M_{n}(s)$. Conversely, and taking into account Figure 4, if $s \neq s^{*}$ then we assume that $K_{s}(\sigma)$ varies slightly along $T_{S}$, except when $T_{S}$ cuts across $T_{s^{*}}$. The selection of the minimum over $T_{S}$ of $\left\|\nabla K_{*}^{n}(\sigma)\right\|_{2}$ then implies a low magnitude of $M_{n}(s)$.

- The above assumptions need to be checked only approximately and locally; the approximation should be sufficient to discriminate a local minimizer $s^{* \times} \neq s^{*}$ (low value of $M_{n}\left(s^{* \times}\right)$ ) from the global minimizer $s^{*}$ (high value of $M_{n}\left(s^{*}\right)$ ).

- The assumption of discontinuity of $K_{*}(\sigma)$ all along $T_{s^{*}}$ implies that $\lim _{n \rightarrow \infty} M_{n}\left(s^{*}\right)=+\infty$. This answers the question of defining what is a high value of $M_{n}\left(s^{* *}\right)$ : in practice, a graph of Dirac type is expected for $M_{n}(s)$. 
- The minimum considered in the calculation of $M_{n}(s)$ is taken over $T$ which is onedimensional, corresponding to a number $2 n_{0}$ of calls to $\nabla K_{*}^{n}(\sigma)$ (number of discretization points of the tangents), actually less than the number $n=\frac{1}{2} n_{0}\left(n_{0}+1\right)$ of calls to $J$ or $L$ for a sorting algorithm. It is assumed that a rough calculation of $\nabla K_{*}^{n}(\sigma)$ requires three calls to $K_{*}^{n}(\sigma)$.

The use of criterion $M_{n}$ to test the globality of a minimizer obtained by a descent algorithm is illustrated in Figures 7 and 8 . Figure 7 gives the graph of $M_{n}$ in perspective and transparency. Figure 8 shows the contour lines of $M_{n}$ in $S$ superimposed on the basins $B_{1}, B_{2}, B_{3}$. We verify with this application that, from the four minimizers of $J$, only $s^{*}$ gives a high value for $M_{n}$, namely 1.21 for $s^{*}$ against values of order $10^{-4}$ for a generic point of $S$ in our simulation. However, some numerical side effects can affect the good localization of the maximum of $M$ that characterizes $s^{*}$, see Figures 7 and 8 .

\section{Conclusion}

We have presented an inverse problem and we have justified the method of complex temperatures for determining the two unknown frontiers of a composite rod made of three materials which undergoes heat conduction. This involves two periodic heating fluxes $\phi_{1}$ and $\phi_{2}$ imposed at both ends together with two temperature measurements at the same ends. The method used is valid without further complication if we have a distributed heating source $\phi$ along the rod. In fact, with or without a distributed source, all combinations of either one or both end fluxes, together with all compatible boundary conditions, namely Dirichlet, Neumann and Robin conditions, are possible. In each case, the simulations that were carried out provided similar results.

We have shown that the redundancy of the temperature measurements, obtained using the complex temperatures method, can be used to improve the application of the classical least-squares method to our problem. For this, we have proposed new criteria of a topological nature that suggest the possibility of an optimization approach that is more global. However, their thorough study and efficient implementation remain to be done in the future.

In a certain way, the one-dimensional inverse problem considered in this paper is probably the simplest one in order to bring out the interest of complex temperatures for constructing global optimization tools. In this case, the practical interest of the corresponding results is weakened by the possibility of a direct -but rough-graphical resolution. This is no longer the case at greater dimensions for $n$-composite materials. Also, the perspective of extending these global optimization tools to $n$-composite materials should open fields of practical application, such as photothermal depth profiling.[26-28]

\section{References}

[1] Autrique L, Perez L. On the use of periodic photothermal methods for material diagnosis. Sensors Actuat. B. 2009;135:478-487.

[2] Perez L, Autrique L. Robust determination of thermal diffusivity values from periodic heating data. Inverse Probl. 2009;25:045011.1-045011.21.

[3] Choulli M. Une introduction aux problémes inverses elliptiques et paraboliques. Vol. 65, of Mathématiques et applications. Dordrecht: Springer; 2009. 
[4] Benabdallah A, Gaitan P, Le Rousseau J. Stability of discontinuous diffusion coefficients and initial conditions in an inverse problem for the heat equation. SIAM J Control Optim. 2007;46:1849-1881.

[5] Henrot A, Pierre M. Variation et optimisation de formes. Vol. 48, in Mathématiques et applications. Berlin: Springer; 2005.

[6] Delfour MC, Zolsio JP. Shapes and geometries: metrics, analysis, differential calculus, and optimization. Advances in design and control. 2nd ed. Philadelphia (PA): SIAM; 2011.

[7] Engl HW, Hanke M, Neubaeur A. Regularization of inverse problems. Dordrecht: Kluwer; 1996.

[8] Kazemzadeh Parsi MJ, Daneshmand F. Solution of geometric inverse heat conduction problems by smoothed fixed grid finite. Finite Elem. Anal. Des. 2009;45:599-661.

[9] Gonzalez M, Goldschmit MB. Inverse geometry heat transfer problem based on a radial basis functions geometry representation. Int. J. Numer. Meth. Eng. 2006;65:1243-1268.

[10] Lesnic D, Bin Mohsin B. Inverse shape and surface heat transfer coefficient identication. J. Comput. Appl. Math. 2012;236:1876-1891.

[11] Marin L, Elliot L, Ingham DB, Lesnic D. The boundary element method for the numerical recovery of a circular inhomogeneity in an elliptic equation. Eng. Anal. Bound. Elem. 2004;28:413-419.

[12] Mera NS, Elliot L, Ingham DB. Numerical solution of a boundary detection problem using genetic algorithms. Eng. Anal. Bound. Elem. 2004;28:405-411.

[13] Karageorghis A, Lesnic D. Application of the MFS to inverse obstacle scattering problems. Eng. Anal. Bound. Elem. 2011;35:631-638.

[14] Hohage T, Rapun ML, Sayas FJ. Detecting corrosion using thermal measurements. Inverse Probl. 2007;23:53-72.

[15] Zhao H, Yan L, Lin J. On the interface identication of free boundary problem by method of fundamental solution. Numer. Linear Algebr. Appl. 2013;20:385-396.

[16] Kamynin LI. Existence of boundary-value problem solution for a parabolic equation with discontinuous coefficients. Izv AN SSSR Ser Mat. 1964;28:721-744.

[17] Lions JL, Magenes E. Non-Homogeneous boundary value problems and applications. Vol. II, Berlin: Springer-Verlag; 1972.

[18] Dautray R, Lions JL. Analyse mathématique et calcul numérique pour les sciences et les techniques. Vol. 8. Paris: Masson; 1988.

[19] Brezis H. Analyse fonctionnelle: théorie et applications. 2nd ed. Paris: Masson; 1987.

[20] Rudin W. Functional analysis. 2nd ed. New York (NY): McGraw-Hill, Inc; 1995.

[21] Yosida K. Functional analysis. Berlin: Springer; 1965.

[22] Zauderer E. Partial differential equations of applied mathematics. 3rd ed. Hoboken (NJ): WileyInterscience; 2006.

[23] Alifanov OM, Artyukhin EA, Rumyantsev SV. Extreme methods for solving Ill-posed problems with application to inverse heat transfer problems. New York (NY): Begell House Inc; 1995.

[24] Hartman P. Ordinary differential equations. 2nd ed. Vol. 25. Philadelphia (PA): SIAM; 2002.

[25] Jolly JC. A gradient calculus and a numerical scheme for a nonlinear inverse heat conduction problem. In: 5th German polish conference on optimization methods and applications[GPCO'2011]; Dobczyce; Poland; 2011.

[26] Glorieux C. Li Voti R, Thoen J, Bertolotti M, Sibilia C. Photothermal depth profiling: analysis of reconstruction errors. Inverse Probl. 1999;15:11-49.

[27] Chen ZJ, Zhang SY. Thermal depth profiling reconstruction by multilayer thermal quadrupole modeling and particle swarm optimization. Chin. Phys. Lett. 2010;27:026502.1-026502.4.

[28] Chen ZJ, Fang JW, Zhang SY. Photothermal depth profiling for multilayered structures by particle swarm optimization. J. Phys. Conf. Ser. 2011;278:012033.1-012033.4. 